\title{
Optimizing linear phased array transducers for non-destructive evaluation of thin layers
}

\section{International Review of Applied Sciences and Engineering}

$11(2020) 2,147-156$

DOI:

$10.1556 / 1848.2020 .20022$

(C) 2020 The Authors

\section{ORIGINAL RESEARCH PAPER}

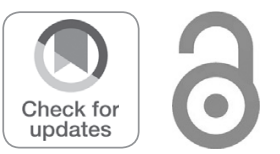

*Corresponding author.

E-mail: mariam.achbal@gmail.com

\author{
MARIAM ACHBAL ${ }^{1 *}$ (1), ABDELLATIF KHAMLICHI ${ }^{2}$ (1) and \\ FADOUA EL KHANNOUSSI ${ }^{2}$ \\ ${ }^{1}$ Department of Physics, Faculty of Sciences, Tetouan, Morocco \\ ${ }^{2}$ Department of Industrial and Civil Sciences and Technologies, National School of Applied Sciences, \\ Tetouan, Morocco
}

Received: August 05, 2019 - Accepted: November 03, 2019

Published online: June 30, 2020

\section{ABSTRACT}

In this work, a numerical method is proposed in order to achieve design optimisation of phased array (PA) probes for the special application of defects detection in thin films. This approach relies on an extended Fourier-based model that was adapted to predict the two-dimensional ultrasonic displacement field taking place in a thin plate under individual excitation of PA probe elements which have arbitrary orientation with respect to the examined part surface. Excitation is applied through a fluid couplant and is operated at scheduled delays that are managed to enable emission of constructive pulses. This gives the possibility to steer sound waves towards a direction and to focalize the beam in a selected point. An optimisation algorithm based on the concept of pattern search that does not require evaluation of a gradient was used to find the best match in the multidimensional analysis space of possibilities including the elements orientation angles, the elements lengths, the inter-elements distances and work frequency. Optimisation was performed with the objective to maximize the displacement amplitude at the focal point while minimizing simultaneously the effect of beam side lobes. The results obtained by this approach reveal that focalisation can be achieved with enhanced features in comparison with previous algorithms assuming linear elements that are parallel to the surface of the plate.

\section{KEYWORDS}

phased array, ultrasounds, optimisation, thin layers

\section{INTRODUCTION}

The principle of phased array (PA) elements had emerged at first in radar system applications [1]. Attempts to apply ultrasonic PAs-based technique for non-destructive testing of structures were made as early as in 1954 [2]. Besides, ultrasonic PAs have been largely applied in the field of medical diagnostics [3], in particular for echocardiography to perform localization of pericardial effusion [4]. Ultrasonic PAs have regained interest beginning from the late 1990s and turned to be a well-established technology that is suitable for non-destructive evaluation (NDE) of structures [5, 6]. During the last two decades, more progress has been continuously achieved in the design of these highly accurate inspection devices.

A PA probe consists of integrated multi-elements which are individually excited in order to emit constructive pulses at scheduled delay times. These delays allow for dynamic controlling of ultrasound beam in terms of beam steering and focussing, resulting in a more flexible manoeuvrability of testing practice [7]. Inspection can then be monitored more straightforwardly by performing, electronically based, sweeping of the ultrasound beam in the area of interest without needing to move considerably the probe.

The pattern of the beam field radiated from an ultrasonic linear PA depends on the transducer characteristics such as frequency, number of elements, pitch, aperture size and element width. These parameters should be fixed cautiously in order to circumvent the 
existence of spurious side lobes with high amplitudes which deteriorate the image quality. To achieve high effectiveness in the field of PAs application in NDE, the transducer should be designed in conformity of a given configuration of testing materials [8]. Considering linear PAs, the spatial pressure distribution of the emitted ultrasonic waves that determine the beam steering characteristics was analysed in ref. [9]. The effect of array element size was studied in ref. [10]. The influence of the intervening array parameters such as interelement spacing, wavelength and number of elements on the beam directivity was investigated in ref. [11]. The aim was to realize optimum efficiency of beam steerability.

The optimisation of PAs design requires simulation tools that have the capacity to enable predicting radiation beam patterns from array transducers with enough accuracy. A series of important works that have been performed in the field of calculation of ultrasonics beams generated by appropriate transducers can be found in the literature. The simulation of the beam field as radiated from a single element transducer can be carried out by using the Rayleigh-Sommerfeld Integral (RSI) approach [12,13]. For complex geometries and complex materials, the Gaussian beams and multi-Gaussian beams (MGB)-based methods were derived [14-16]. These models admit the paraxial approximation of wave propagation, which assumes that ultrasonic bulk waves produced by transducers take the form of well-collimated beams. They were found to achieve both high computational efficiency and accuracy.

Park et al. [17] developed a beam-like model for the particular case of linear array transducers by assuming that the resulting ultrasound signal from this device can be approximated by a superposition of MGB [15]. They evaluated accuracy of this model by comparing the obtained results to the solution provided by RSI-based method. Because the MGB model is an outcome of the paraxial approximation, the model developed by Park et al. showed satisfactory results for a steering angle that remains limited to $20^{\circ}$. To overcome these restrictions, Huang et al. [18] proposed a new approach of using Gaussian beams in modelling the PA transducers. This approach called the multi-Gaussian array (MGA) beam model is based on introducing two modifications to the fundamental MGB model. The first modification consists in applying a linear phasing sequence to the elements of an array, and the second one is set by multiplying a linearly phased MG beam model by a correction factor taken to the missing directivity function. The predictions made by the MGA beam model were found to be in good agreement with the results obtained by the more exact RSI model, while the MGA beam model was recognized to be computationally far more superior than this last.

Zhao et al. [19] developed a non-paraxial multi-Gaussian beam (NMGB) model they used to calculate the beam fields radiated by a linear PA transducer. Contrary to the paraxial MGB model, the distance factor was expanded with additional approximation terms in order to achieve more accuracy beyond the region where paraxial approximation is valid. The accuracy provided by this model was tested through comparisons based on both numerical and experimental studies. NMGB model was recognized to yield an effective numerical method for fast determination of the displacements associated to the propagation of ultrasound waves. However, this approach suffers from the fact that the strain, which is given by the spatial derivatives of the displacement vector, remains to be determined by using an appropriate numerical differentiation method. This operation consumes extra time when the rough discrete outputs of the NMGB model are utilized directly and the obtained strains would be calculated with some numerical errors. This is why a Fourier-based model [20] appears to be more suitable for dealing with problems involving the evaluation of strains. These can be advantageously represented in Fourier approach by analytic expressions, which enable then their accurate and rapid computation. Using the Fourier approach, Wang et al. [20, 21] have developed a straightforward model for dealing with the analysis of diffraction that occurs following the emission of ultrasonic waves by PAs transducers. This consists of a simple analytic expression that gives explicitly the displacement field caused by the ultrasonic wave propagation in the tested medium. Predictions of this model were compared to the NMGB results and were found to be in excellent agreement to them.

Use is made in this work of the Fourier-based model in order to predict the two-dimensional ultrasonic displacement field taking place in a thin plate as generated by means of an appropriate firing protocol of the elements composing a linear PA transducer coupled to the surface of the plate by a fluid couplant. Focus is on the problematic of generation of ultrasonic beams over a wide range of steering angles. This is important for practical application of the phased array ultrasonic testing (PAUT) in the field of films that have the form of elastic plates with relatively small thickness. As focalisation of a beam cannot be easily performed by elements that remain parallel to the plane surface of the inspected part, angular orientation of these elements is considered in order to direct the rays with high effectiveness towards the desired target point in the panel. The elements need also to be coupled to the inspected part by a solid angle wedge which lays directly on the fluid couplant to ensure appropriately the connection of the element to the part. The array parameters of the inspection conditions will be determined through an optimisation procedure which intends maximizing the amplitude of displacement at the focal point while minimizing the effect of side lobes. The primary advantage of the proposed design procedure is that it provides a systematic methodology for PAUT probes optimisation. This offers also the possibility to control more flexibly the steering of the beam towards the target point fixed in the inspected structure. It enables moreover avoiding costly prototyping before the final stage of micro-fabrication of the probe $[22,23]$.

It should be noticed that in the actual work, the surface of the inspected part is assumed to be perfectly smooth and the roughness effect is neglected. It was stated in the literature that, depending on the statistical characteristics of real surfaces such as arithmetic average heights, roughness can induce additional attenuation as well as it yields increase of noise [24]. In the particular case of PAUT probes configurations, the effect of roughness is expected to perturb focalisation of ultrasonic waves and to decrease resolution of 
the steering beam. The attenuation effect could be taken into account by augmenting damping of the element radiated beam, while the noise effect requires performing a refined description of the coupling between the transducers and the rough surface of the inspected part.

The structure of this article is as follows. In Section 2, modelling of the PAs inspection conditions by means of the Fourier-based method [20] is performed. Then the technique of focussing an ultrasonic wave beam to attain more efficiently a focal point is detailed. This will be followed by considering the probe design problem that consists of finding the best angular direction of the elements with respect to the surface normal of the examined part. After that, integrated angle wedges used for coupling the inclined elements to the investigated part will be studied. In the third section, the array parameters will be determined as solution of mathematical program by using the Matlab command patternsearch. The results of the optimisation problem will be finally presented in the fourth section.

\section{MODELLING LINEAR ARRAY TRANSDUCER}

The present section is aimed at developing a model of ultrasonic beam generation in a thin plate medium by a linear array transducer towards monitoring its steering and focalisation. The radiation pattern of ultrasonic PA can be obtained through the superposition of beam fields generated by all the transducer elements and shifting the time delay to be applied to each element for the proper beam steering and/or focussing.

In the following, use is made of the mathematical formulation based on Fourier approach that was used by Wang et al. $[20,21]$ to analyse steering for ultrasonic wave patterns in solids generated by PA transducers. This modelling is appropriate in the situation where the array element is assumed to be in contact directly with a solid through a fluid couplant. The boundary conditions correspond then to a pressure uniformly distributed over the element surface. This pressure generates multiple waves such as dilatational waves and shear waves propagating in the bulk of the solid as well as surface waves. For frequencies sufficiently high, the dilatational waves can be modelled by Rayleigh-Sommerfeld like integral yielding then to Fourier-based model. Considering linear PA configuration, the problem is two dimensional with transducers assumed to be infinitely long along their direction. The geometry of the medium is presented in Fig. 1.

The 2D displacement of the focal point $P(x, z)$ in the half plane elastic medium $z>0$ is given, for longitudinal ultrasonic waves, by the following expression [20].

$\vec{u}(x, z)= \begin{cases}-\sum_{m=1}^{M} \frac{\vec{U}_{m}}{i_{m}} \frac{\exp \left(i K_{m} b_{u}\right)-\exp \left(i K_{m} b_{l}\right)}{x-x_{c m}} & \text { for } x \neq x_{c m} \\ 2 \sum_{m=1}^{M} \vec{U}_{m} \frac{a_{m}}{\sqrt{z^{2}+a_{m}^{2}}} \exp \left(i K_{m} \sqrt{z^{2}+a_{m}^{2}}\right) & \text { for } x=x_{c m}\end{cases}$

with

$$
\begin{gathered}
\vec{U}_{m}=\frac{p_{m}}{2 \pi \rho c_{l m}^{2}} D\left(\theta_{p}\right) \vec{d}_{p m} \exp \left(2 i \pi F_{m} \Delta \tau_{m}\right) \\
b_{l}=\sqrt{z^{2}+\left[x-\left(x_{c m}-a_{m}\right)\right]^{2}} \\
b_{u}=\sqrt{z^{2}+\left[x-\left(x_{c m}+a_{m}\right)\right]^{2}} \\
K_{m}=\kappa_{m}+i \xi_{m}
\end{gathered}
$$

where $M$ is the number of transducers, $x_{c m}$ the coordinate along the $x$-axis of the centre of the $m$ th transducer, $a_{m}$ the half-width of the $m$ th transducer, $\rho$ is the density of the medium, $p_{m}$ the pressure exerted by the $m$ th transducer, $c_{l m}$ the longitudinal wave speed which depends on the work frequency of the $m$ th transducer, $\theta_{P m}$ is the angle between the line joining the centre of the $m$ th transducer to point $P$

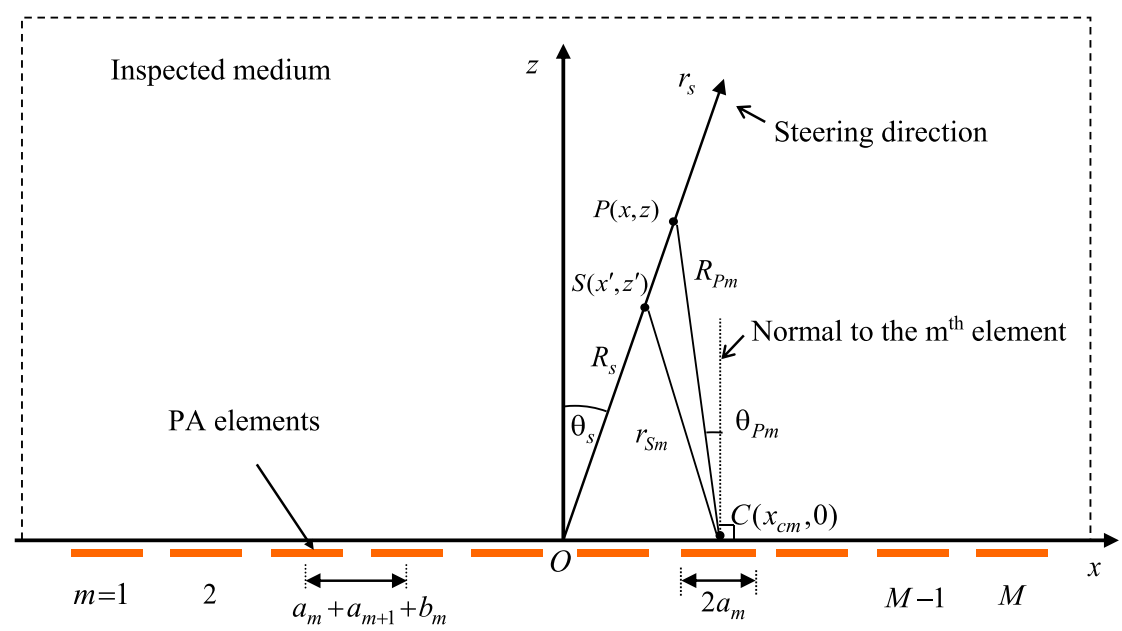

Fig. 1. Configuration of PA transducer for ultrasonic beam steering at the surface of the inspected solid; the steering angle is measured from the centre of the PA, $P$ is the focal point position and $C$ is the centre of an array element 
and this $m$ th element surface normal at its centre $x_{c m}, \theta_{s}$ the steering angle which is assumed to be positive when measured from the $\mathrm{z}$-axis in the clockwise direction, $R_{s}$ is the polar distance measured from the point $O$ to a point $S$ located on the steering direction and $\xi_{m}$ the attenuation coefficient for the $m$ th transducer. The wave number $\kappa_{m}$ is given by $\kappa_{m}=\omega_{m} / c_{l m}$ where $\omega_{m}=2 \pi F_{m}$ is the angular frequency of the waves emitted by the $m$ th transducer.

In Eq. (2), the directivity function associated to the $m$ th transducer is given by:

$$
D\left(\theta_{P m}\right)=\frac{c_{m}^{2}\left[c_{m}^{2}-2 \sin ^{2}\left(\theta_{P m}\right)\right] \cos \left(\theta_{P m}\right)}{2\left[2 \sin ^{2}\left(\theta_{P m}\right)-c_{m}^{2}\right]^{2}+\sin ^{2}\left(2 \theta_{P m}\right) \sqrt{c_{m}^{2}-\sin ^{2}\left(\theta_{P m}\right)}}
$$

with $c_{m}=c_{l m} / c_{t m}$ where $c_{t m}$ is the longitudinal shear wave speed of the $m$ th transducer.

As in practice $c_{m}>1$, Eq. (6) shows that the directivity factor is essentially affected by the factor $\cos \left(\theta_{P m}\right)$. This is in contrast with the case of pure acoustic waves in which the directivity factor is dependent on the element width, the wave length and the angle measured from the element normal [15]. Even if this dependency weakens when the element width is comparable to the acoustic wave length, dependency on the angle measured from the element normal remains. This shows the main advantage of introducing a fluid couplant to monitor wave propagation into the bulk solid. The directivity factor, for a given element of the array, will then be function of only the element centre position through the angle $\theta_{P m}$ as show in Eq. (6).

The polarization vector for the $m$ th transducer is given by:

$$
\vec{d}_{p m}=\frac{x-x_{c m}}{R_{P m}} \vec{e}_{x}+\frac{z}{R_{P m}} \vec{e}_{z}
$$

with $R_{P m}$ representing the distance separating the focal point $P(x, z)$ and the centre of the $m$ th transducer, which is given by:

$$
R_{P m}=\sqrt{\left(x-x_{c m}\right)^{2}+z^{2}}
$$

The time delay for an ultrasonic wave arriving at a point $S\left(x^{\prime}, z^{\prime}\right)$ belonging to the steering direction fixed by $\theta_{s}$ and emitted from the point $C\left(x_{c m}, 0\right)$, with regards to that emitted from the origin $O(0,0)$, is shown to be

$$
\Delta \tau_{m}=\frac{R_{s}-\sqrt{R_{s}^{2}+x_{c m}^{2}-2 R_{s} x_{c m} \sin \theta_{s}}}{c_{l m}}
$$

Equations (1)-(9) are used hereafter to simulate the displacement field in the medium according to Fourier model [20].

\subsection{Orientation angle}

In the particular case of thin films, the thickness $h$ of the plate can be very small in comparison with the distance between the centre of the PA transducer and the desired focal point $P$. Considering the reference axes $(O, x, y)$, the $z$-coordinate of the focal point is given by $z_{P}=$ $R_{P m} \cos \theta_{P m}$ and the $x$-coordinate is $x_{P}=x_{C m}-R_{P m} \sin$
$\theta_{P m}=x_{C m}-\sqrt{R_{P m}^{2}-z_{P}^{2}}$. To reach focal points that are far from the PA centre, the polar radius $R_{P m}$ should be large. This can be achieved only by means of polar angles for which $\theta_{P m} \rightarrow \pi / 2$ or equivalently $\cos \theta_{P m}$ is small. The angle between the normal to the element and the straight line joining the centre of the element and the focal point should be high in order to reach points that are close to the part surface. This limits performance as, according to the expression of $D\left(\theta_{P m}\right)$, the amplitude which is proportional to $\cos \theta_{P m}$ will be reduced for high values of $\theta_{P m}$, leading then to huge loss of wave power during the focalisation operation. Fig. 2 illustrates, for a material for which the Poisson's coefficient is equal to 0.3 , the variations of the directivity function in terms of the angle between the normal and the focalization direction. As shown in this figure, for wave incidence exceeding $1 \mathrm{rad}$, the value of directivity factor is well decreased in comparison to its maximum value which is equal to 0.5 . The ultrasonic beam generated by a planar linear transducer propagating through a planar interface will then have limited effectiveness in investigating plates if the orientation of the element direction is not changed.

To overcome this situation, it is proposed in the actual work to give the PA elements an initial orientation angle $\varphi_{m}$ which is intended to improve the directivity of the propagating beam, see Fig. 3. Using oriented elements by adding the angle $\varphi_{m}$ enables to monitor more suitably ultrasonic beam directivity towards a point $S$ located on the steering direction and defined by the polar coordinates $\theta_{s}$ and $R_{s}$. This enhances focalisation of waves with intense amplitudes through that point and resolution of defect imagining testing can be drastically improved.

According to Fig. 3, the angle between the line joining the centre of the $m$ th transducer to the focal point $P$ and the $m$ th transducer surface normal at its centre position is obtained as

$$
\theta_{P m}=\varphi_{m}-\tan ^{-1}\left(\frac{x_{c m}-x}{z}\right)
$$

The orientation angle can be fixed by imposing the normal to the element at its centre to be pointing towards a

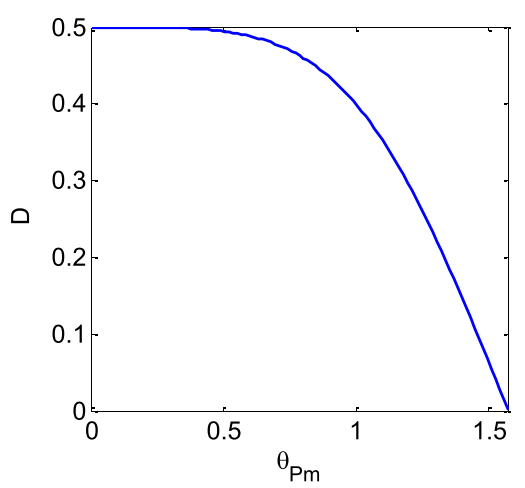

Fig. 2. Variation of directivity factor as function of the angle between the normal and the focalization direction; Poisson's coefficient $\nu=0.3$ 


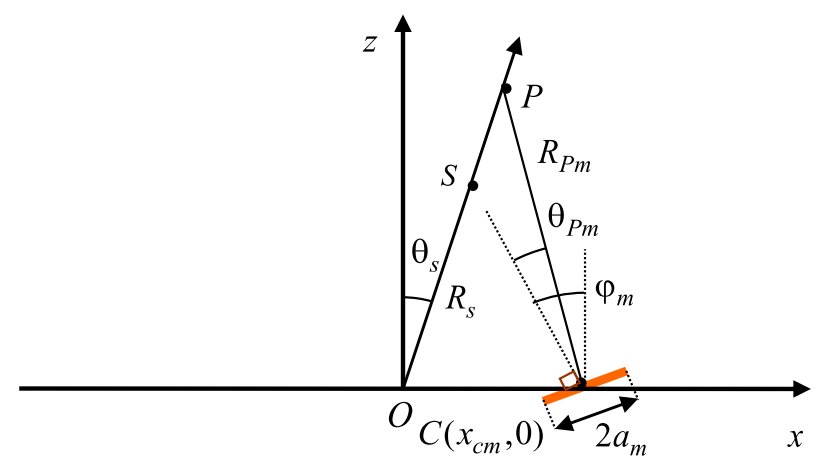

Fig. 3. Geometry of the $m$ th element with its normal rotated by angle $\varphi_{m}$

point $S$ located on the steering direction, in this particular case the direction of the $m$ th element is fixed by

$$
\varphi_{m}=\tan ^{-1}\left(\frac{x_{c m}-R_{s} \sin \theta_{s}}{R_{s} \cos \theta_{s}}\right)
$$

with $\left.\varphi_{m} \in\right]-\pi / 2, \pi / 2[$.

Equations (10) and (11) are used to calculate the orientation angle of each element in the array transducer. The orientation angles $\varphi_{m}$ are included in the list of design parameters of the PAs transducer and will be subject to the optimisation problem aiming at maximising the wave amplitude near the desired focal point.

\subsection{Angle beam wedge transducer}

An inclination of the array elements was proposed to improve the efficiency of detection of defects in plates with small thickness. However, to give the elements an initial angle, we need to use integrated wedges for coupling each element of the array transducer to the surface of the inspected part through a fluid couplant. In this case, each element is considered as an angle beam transducer. This transducer consists of a contact $\mathrm{P}$-wave probe mounted at an angle on a solid wedge and this wedge is in planar contact with the plate to be inspected through a fluid couplant as shown in Fig. 4.

The wedge transducer in assumed to be in smooth contact with the surface, meaning that the shear stress will be assumed to vanish at the interface. This will not be the case if the contact surface is rough with roughness scale being comparable to the actual wave length. Fig. 5 describes the proposed design of array transducer with integrated wedges. As mentioned above, the global radiation beam pattern emitted from the PA transducer elements can be

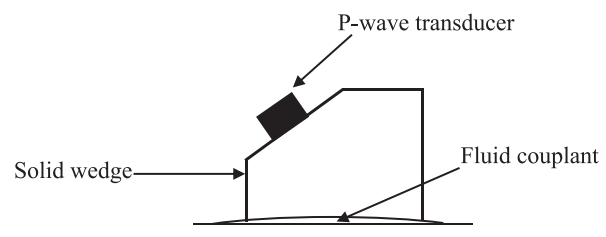

Fig. 4. Angle beam transducer

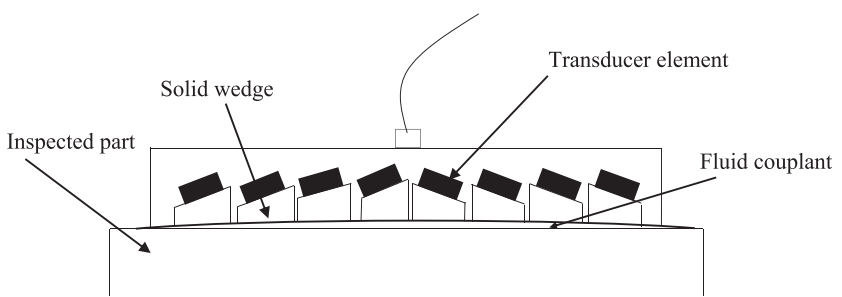

Fig. 5. Linear array probe with integrated wedges

considered as a superposition of individual beam patterns monitored by suitable time delays.

Considering, in the case of a single beam wedge transducer, a harmonic incident P-wave of angular frequency $\omega$ that hits the interface separating the wedge from the inspected part at incident angle $\theta_{p 1}$, the generated P-waves and $\mathrm{S}$-waves write as given by ref. [13].

$$
\begin{aligned}
& \phi_{\text {inc }}=A_{i} \exp \left[i k_{p 1}\left(x \sin \theta_{p 1}+z \cos \theta_{p 1}\right)-i \omega t\right] \\
& \phi_{\text {reft }}=A_{r} \exp \left[i k_{p 1}\left(x \sin \theta_{p 1}-z \cos \theta_{p 1}\right)-i \omega t\right] \\
& \phi_{\text {trans }}=A_{t} \exp \left[i k_{p 2}\left(x \sin \theta_{p 2}+z \cos \theta_{p 2}\right)-i \omega t\right] \\
& \psi_{\text {reftl }}=B_{r} \exp \left[i k_{s 1}\left(x \sin \theta_{s 1}-z \cos \theta_{s 1}\right)-i \omega t\right] \\
& \psi_{\text {trans }}=B_{t} \exp \left[i k_{s 2}\left(x \sin \theta_{s 2}-z \cos \theta_{s 2}\right)-i \omega t\right]
\end{aligned}
$$

where $t$ is time, $\theta_{p 2}$ is the refracted angle of $\mathrm{P}$-wave, $\theta_{s 1}$ is the reflected angle of S-wave, $\theta_{s 2}$ is the refracted angle of S-wave, $k_{p 1}$ the incident wave number, $k_{p 2}$ is the refracted wave number of $\mathrm{P}$-wave, $k_{s 1}$ is the reflected wave number of Swave, $k_{s 2}$ is the refracted wave number of S-wave, $A_{i}$ is the incident amplitude of $\mathrm{P}$-wave, $A_{r}$ is the reflected amplitude of P-wave, $A_{t}$ is the transmitted amplitude of P-wave, $B_{r}$ is the reflected amplitude of S-wave, $B_{t}$ is the transmitted amplitude of S-wave. The subscript 1 is relative to the wedge material, while the subscript 2 is relative to the inspected material.

Due to fluid coupling, the boundary conditions written on the interface $z=0$ take the form:

$$
\begin{gathered}
\left(u_{z}\right)_{1}=\left(u_{z}\right)_{2} \\
\left(\tau_{z z}\right)_{1}=\left(\tau_{z z}\right)_{2} \\
\left(\tau_{x z}\right)_{1}=0 \\
\left(\tau_{x z}\right)_{2}=0
\end{gathered}
$$

where $\tau_{z z}$ is the normal stress component to the interface and $\tau_{x z}$ is the shear stress acting at the interface.

Applying the boundary conditions given by Eqs. (17)(20) and phase matching for all the terms on the boundary, $z=0$ gives the following generalized Snell's law

$$
\frac{\sin \theta_{p 1}}{c_{p 1}}=\frac{\sin \theta_{s 1}}{c_{s 1}}=\frac{\sin \theta_{p 2}}{c_{p 2}}=\frac{\sin \theta_{s 2}}{c_{s 2}}
$$

For angle beam transducers, the incident wave angle influences the nature of the wave existing in the second medium. For small incident angles that verify $\theta_{i}<\sin ^{-1}\left(c_{p 1} / c_{p 2}\right)$, 
the reflection and transmission coefficients are real. This means that following reflection or transmission the waves are plane waves with amplitudes that are proportional to the incident plane wave. In this case, both transmitted $\mathrm{P}$ - and S-waves are present in the inspected medium. When the incident wave exceeds the critical angle $\left(\theta_{c r}\right)_{1}=\sin ^{-1}\left(c_{p 1} / c_{p 2}\right)$, the waves obtained through reflection and transmission are combined in an inhomogeneous shear transmitted wave that propagates into the second medium along the interface with exponential decay of amplitude as function of depth from the interface. If the incident angle exceeds a second critical angle $\left(\theta_{c r}\right)_{2}=\sin ^{-1}\left(c_{p 1} / c_{s 2}\right)$ which exists only when $c_{s 2}<c_{p 1}$, the transmitted waves travelling in the interface of the second material are inhomogeneous P- and S-waves [13].

As in the present work, generation of longitudinal waves in the inspected film is of first interest, the limitation imposed on the incident angle is considered in the optimization problem.

The reflected $\mathrm{P}$ - and $\mathrm{S}$-waves in the wedge could pass into the panel after multiple reflections. This could produce noisy interference which may affect the response of the transmitted waves, complicating the interpretation of the received signal following scattering at flaws. In order to avoid this situation, several methods are used in practice with the aim of minimizing the reflections. Among the proposed solutions, the geometry of the wedge can be made long to allow the wave path to escape from the wedge, the wedge surface can be grooved to scatter the reflected waves in many directions and an absorbing material can be added to eliminate further reflections [13].

\section{OPTIMIZATION OF THE TRANSDUCER}

In this section, we aim to find the optimal design of the PA transducer for improving the detection of defects in thin plates. By using the ultrasonic displacement equation defined in the first section, an optimisation procedure has been conducted to determine the array design parameters. In the present study, it is suggested to select the transducer parameters with the purpose of both maximizing the amplitude of displacement in the focussing point and minimizing the harmful effect of side lobes. These requirements are integrated effectively by appropriate definition of the cost function. The obtained optimization problem is solved by using the Matlab command patternsearch [25]. Bound constraints are also specified in order to stabilize solution procedure provided by this command.

\subsection{Design variables}

The optimisation process is performed based on the model of PA described in the first section. This modelling uses the Fourier method to predict the generated ultrasonic beam pattern through Eq. (1). In order to find the optimum beam directivity according to the criteria mentioned above, the parameters defining the PA device configuration should be taken as basic variables in the context of an optimisation problem. Several parametric studies were realised in order to assess the relative effect of design parameters on the results. These were performed by varying the number of elements, the focal point position, the steering angle, the inter-element spacing, the element width, the level of pressure activating the transducer and the frequency bandwidth [26]. It was found that these parameters impact the beam steering and the directivity of the propagating beam in different ways.

Within the systematic approach to PA probe optimization considered in this work, several key basic variables were retained. Optimization of design of the PA transducer was conducted in terms of the following geometric and mechanical properties: the number of transducer elements $M$, the frequencies $F=\left[\begin{array}{llll}F_{1} & F_{2} & \ldots & F_{M}\end{array}\right]$, the half-width of the transducer elements $A=\left[\begin{array}{llll}a_{1} & a_{2} & \ldots & a_{M}\end{array}\right]$, the interelements distance $B=\left[\begin{array}{llll}b_{1} & b_{2} & \ldots & b_{M-1}\end{array}\right]$ and the elements orientation angle $\Phi=\left[\begin{array}{llll}\varphi_{1} & \varphi_{2} & \ldots & \varphi_{M}\end{array}\right]$.

Note that the PA probe is a linear system, so the pressure $p_{m}$ activating the $m$ th element produces a pure proportional effect. This parameter which is related to the power of the transducer element was discarded from the list of active design variables. Otherwise, the optimization process would have provided the obvious solution associated to the maximum possible value of $p_{m}$.

To avoid grating lobes [8], the following Shannon like constraint should be fulfilled

$$
2 \times \max _{m=1}^{M}\left(a_{m}+a_{m+1}+b_{m}\right)<\min _{m=1}^{M}\left(\lambda_{m}\right)
$$

with $\lambda_{m}=\frac{c_{l m}}{F_{m}}$ designates the wave length.

Denoting $\Lambda=\min _{m=1}^{M}\left(\lambda_{m}\right)$ and taking $\beta_{m} \geq 0$, Eq. (22) can be rewritten as

$$
a_{m}+a_{m+1}+b_{m}=\frac{\Lambda}{2+\beta_{m}} \quad \text { for } \quad m=1, \ldots, M
$$

Taking $\alpha_{m} \geq 0$ and putting $a_{m}=\frac{\Lambda}{4+\alpha_{m}}$, one obtains from Eq. (23) the expression for the inter-elements distance as

$$
b_{m}=\frac{2 \alpha_{m}+2 \alpha_{m+1}+\alpha_{m} \alpha_{m+1}+\left(8+\alpha_{m}+\alpha_{m+1}\right) \beta_{m}}{\left(2+\beta_{m}\right)\left(4+\alpha_{m}\right)\left(4+\alpha_{m+1}\right)} \Lambda
$$

The total length of the PA transducer is then

$$
L=2 \sum_{m=1}^{M} a_{m}+\sum_{m=1}^{M-1} b_{m}
$$

Using Eqs. (23) and (24), the length of the PA transducer is obtained as

$$
\begin{aligned}
L= & \Lambda\left(\sum_{m=1}^{M-1} \frac{16+2 \alpha_{m}+6 \alpha_{m+1}+\alpha_{m} \alpha_{m+1}+\left(24+\alpha_{m}+5 \alpha_{m+1}\right) \beta_{m}}{\left(2+\beta_{m}\right)\left(4+\alpha_{m}\right)\left(4+\alpha_{m+1}\right)}\right. \\
& \left.+\frac{1}{4+\alpha_{M}}\right)
\end{aligned}
$$

The effective design variables are now defined by the following set of parameters $\left\{\alpha_{m}, \beta_{m}, \varphi_{m}, F_{m}, M\right\}$ with $m=1, \ldots, \quad M$ except for $\beta_{m}$ where $m=1, \ldots, M-1$. 


\subsection{Definition of the objective function}

The cost function is related to maximizing the displacement amplitude at the focal point, while minimizing dispersion around a given focal point over the distribution of amplitudes along the direction $z=z_{P}=R_{P} \cos \theta_{P}$. Denoting $\vec{u}_{P, n}=\vec{u}\left(x_{n}, z_{P}\right)$, the displacement field restricted on the parallel line to the material surface passing through the focal point: $z=z_{P}$, the standard deviation (SD) $\sigma$ of the norm of displacements $\left\|\vec{u}_{P, n}\right\|$ is used to quantify the amount of dispersion of displacement around this axis. A low value of this measure indicates that the data points tend to be close to the mean, while a high SD means that the data points are spreading out from the mean value. This ensures a highresolution main lobe with reduced effect of side lobes.

Fixing the number of PA elements $M$, the multi-objective cost function is taken to have the following form:

$$
\begin{aligned}
\Psi_{M}\left(\alpha_{m}, \beta_{m}, \varphi_{m}, F_{m}\right)= & (q-1) \frac{\sigma_{r e f}}{\sigma\left(\left\|\vec{u}_{P, n}\right\|\right)} \\
& -q \frac{\left\|\vec{u}\left(x_{P}, z_{P}\right)\right\|}{u_{r e f}}
\end{aligned}
$$

where $\sigma_{\text {ref }}$ designates a reference $\mathrm{SD}, u_{r e f}$ a reference displacement and $q$ is a parameter that is taken in the interval $[0,1]$. This parameter is used to weight the two objectives, in the framework of a passive multi-objective approach. It serves to tune the contributions of the two terms appearing in the right-hand side of Eq. (27). The constants $u_{\text {ref }}$ and $\sigma_{\text {ref }}$ are fixed in order to have the same order of magnitude for the two terms in the second half of Eq. (27).

\subsection{Mathematical program}

Adding bound like constraints, the mathematical program associated to the optimisation problem of PA transducer is written as

$\left\{\begin{array}{l}\text { Minimise } \quad\left\{\Psi_{M}\left(\alpha_{m}, \beta_{m}, \varphi_{m}, F_{m}\right)\right\} \text { under the constraints } \\ 0 \leq \alpha_{m} ; \quad 0 \leq \beta_{m} ; \quad \varphi_{1} \leq \varphi_{m} \leq \varphi_{2} ; \quad F_{1} \leq F_{m} \leq F_{2}\end{array}\right.$

where $F_{1}, F_{2}$ fix the limits of frequency interval of interest and $\varphi_{1}, \varphi_{2}$ are the limits of the elements orientation angle.

Taking the limits on frequency is motivated by the fact that frequency variations should not be too large from one element to another in order that the central frequency could be easily realized by suitable electronics. The values taken by $M$ are here discrete and are taken in the following set $\{8,16,32,64,128\}$ whose members are all exponents of number 2 .

\section{RESULTS AND DISCUSSION}

Solution of the mathematical program defined by Eq. (28) with the cost function given by Eq. (27) in which the amplitudes are explicitly calculated by means of Eqs. (1)-(9) was performed by using the Matlab command patternsearch. The SD was calculated from the obtained displacement amplitudes by using the Matlab command std.

Various simulations that have been conducted by applying the above-mentioned procedure have yielded that the optimum is always achieved with all the parameters $\alpha_{m}$, $\beta_{m}$ and $F_{m}$ being equal. This offers the possibility to further simplifying the mathematical program, which can be written as

$$
\left\{\begin{array}{l}
\text { Minimise } \quad\left\{\Psi_{M}\left(\alpha, \beta, \varphi_{m}, F\right)\right\} \text { under the constraints } \\
0 \leq \alpha ; \quad 0 \leq \beta ; \quad \varphi_{1} \leq \varphi_{m} \leq \varphi_{2} ; \quad F_{1} \leq F \leq F_{2}
\end{array}\right.
$$

where only the orientation angles are varying from one element to another. In this case, the total length of the PA transducer is given by

$$
L=\frac{(4 M-2)+(M-1) \alpha+(6 M-5) \beta}{(2+\beta)(4+\alpha) F} c_{l}
$$

\subsection{Optimal parameters}

Optimisation of PA transducer was conducted by considering a thin plate, modelled as an isotropic material having Young's modulus $E=210 \mathrm{GPa}$, Poisson's coefficient $\nu=0.3$ and density $\rho=7800 \mathrm{~kg} / \mathrm{m}^{3}$. This yields the longitudinal and shear wave velocities for the medium to be respectively $c_{p_{2}}=6020$ and $c_{s}=3218 \mathrm{~m} / \mathrm{s}$. The attenuation coefficient was fixed $\xi_{m}=5 \cdot 10^{-3}$. The pressure applied to the medium by transducer elements was taken to be $p_{m}=10^{6} \mathrm{~N} / \mathrm{m}^{2}$. The simulated plate has width $h=2.5 \mathrm{~cm}$. The focal point was selected with normal incidence $\theta_{S}=0$ at mid-height position $R_{S}=1.25 \mathrm{~cm}$.

Taking the case for which the number of elements is $M=8$, the frequency was bounded by $F_{1}=1 \mathrm{MHz}$ and $F_{2}=1.2 \mathrm{MHz}$, while the orientation angle of elements was constrained to belong to the interval bounded by $\varphi_{1}=-\pi / 3$ and $\varphi_{2}=\pi / 3$. The cost function was fixed by taking $q, \sigma_{r e f}$ and $u_{r e f}$ to be equal respectively to 0.9 , $3.7 \times 10^{-8}$ and $2 \times 10^{-6} \mathrm{~m}$.

Identification of the most advantageous PA probe parameters was performed and has provided the following results: frequency $F=1 \mathrm{MHz}$ with the associated wave number $\kappa_{m}=1043.7 \mathrm{~m}^{-1}$, half width element size $a=1.5 \mathrm{~mm}$, inter-element distance $b=15.7 \mu \mathrm{m}$, optimal orientation angles: $\Phi=\left[\begin{array}{llll}1.05 & 0.976 & 0.725 & 0.583\end{array}\right.$ $-0.583-0.725-0.976-1.05]^{T}$ in rad. Note that the orientation angles are symmetric about the mid-vertical axis of the transducer.

\subsection{Wedges dimensions}

The incident $\mathrm{P}$-wave propagates in the wedge with an angle $\theta_{p_{1}}$ equal to the wedge angle and propagates then in the inspected medium with $\theta_{p_{2}}=\varphi$ as describe in Fig. 6 . 


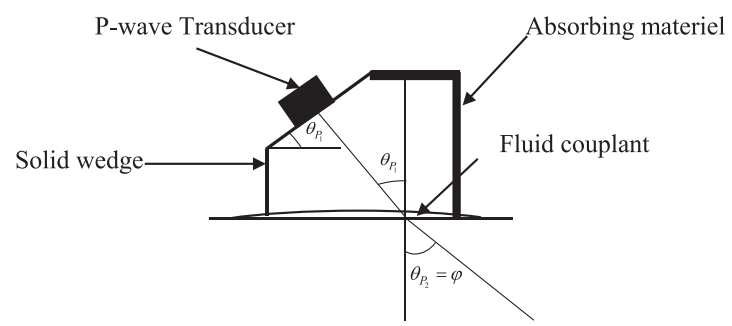

Fig. 6. Angle wedge transducer

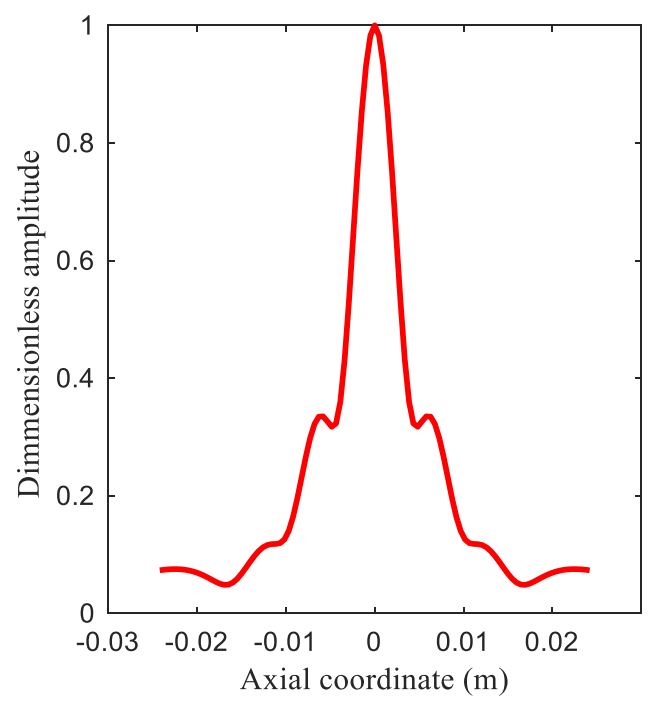

Fig. 7. Dimensionless displacement amplitude over the parallel line through the focal point

As discussed above, the incidence wave angle $\theta_{p_{1}}$ is related to the refraction angle $\theta_{p_{2}}$ by Snell's law through the equation given by:

$$
\sin \theta_{p 1}=\frac{c_{p 1}}{c_{p 2}} \sin \theta_{p 2}
$$

Taking the wedge material to be steel 4,340 , the wave propagates with a longitudinal velocity of $c_{p_{1}}=5850 \mathrm{~m} / \mathrm{s}$. In order to produce a longitudinal wave in the second medium propagating with the optimal angle $\varphi$ presented in Section 4.2 , the angle of the designed wedge for each transducer element should take the values assembled in the following vector: $\Theta=\left[\begin{array}{llll}1.0001 & 0.935 & 0.7006 & 0.6018-0.6018-0.7006\end{array}\right.$ $-0.935-1.0001]^{T}$. Note that all the transducer elements respect the incident wave angle condition, namely $\theta_{1 m}<\sin ^{-1}\left(c_{p 1} / c_{p 2}\right)$ with $c_{p_{1}}=5850$ and $c_{p_{2}}=6020 \mathrm{~m} / \mathrm{s}$.

For the purpose of minimising the part of multiple reflected waves passing into the second medium, an absorbing material can be added to the upper and lateral surfaces of the wedge as shown in Fig. 6.

\subsection{Simulation of the PA transducer}

To evaluate the efficiency of the PA probe with inclined elements compared to the transducers with elements parallel to the surface of the inspected part, simulation was performed by using the propagation wave model described in the first section. The PA probe with inclined elements was taken under the configuration resulting from the optimal design values of the PA transducer as obtained in Section 4.1. Fig. 7 shows the dimensionless amplitude of displacement as function of the axial coordinate on the line passing through the focal point and parallel to the transducer surface for the PA probe with inclined elements. The obtained results have shown a high concentration of the ultrasound beam on the focal point direction with reduced effect of side lobes.

Fig. 8 shows the ultrasonic displacement field as obtained for the two configurations of PA probes. Fig. 8 a presents the ultrasonic displacement field generated by regular array probes with elements parallel the examined part surface. Fig. $8 \mathrm{~b}$ shows the displacement field produced by the PA probe as defined by the optimal design considered in Section 4.1 .

As depicted in Fig. 7, the distribution of displacement amplitude has good directivity and is concentrated in the principal lobe with limited effect from secondary lobes. From Fig. $8 \mathrm{a}$ and b, it can be seen that the proposed optimal model produces superior directivity and higher amplitudes than the regular model with elements oriented in parallel to the part surface. Considerable gain of wave power is achieved through the focalisation operation with inclined elements. This enables producing a high concentration of the

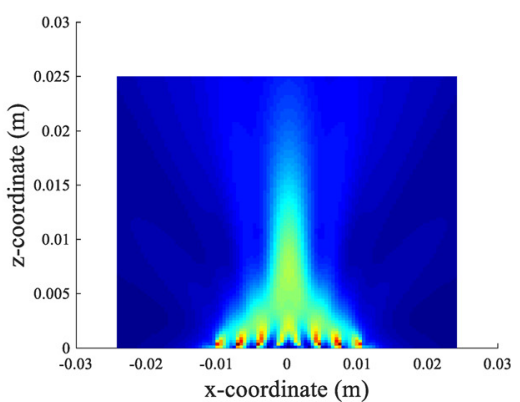

(b)

Fig. 8. Two-dimensional amplitude of the ultrasonic displacement field in the thin plate generated by a. array transducer with elements parallel to the surface $b$. oriented elements transducer 
ultrasonic beam energy in the focal point and insuring a better control of the ultrasound beam.

It should be mentioned that the actual study is purely theoretical and that experimental proof of the obtained results should be made to assess the effectiveness of the findings.

\section{CONCLUSIONS}

In this work, optimal design of PA transducers was achieved for the particular case of film-like plates having thin thickness. After modelling the inspection conditions occurring with focused ultrasonic wave beam generated by multiple elements, the option of giving initial orientations to the elements with respect to the surface of the inspected part was analysed. The pursued objective is increasing concentration of ultrasonic energy just about the focal point while minimizing side lobes effect. Integrated wedges were adopted in order to connect the oriented elements to the plane surface of the plate through a liquid couplant. An adequate optimization procedure which considers angular orientations of the elements directions as basic design variables was proposed. Solution of the obtained mathematical program showed that the optimum is reached with equal mechanical and geometrical parameters of the element except for variable orientation angle from one element to the other. The obtained results have demonstrated the aptitude of the proposed methodology to achieve optimization of PA transducers. Comparison of the results for a transducer with oriented elements and regular planar probe has shown superior concentration of the beam energy in the focal point. It should be essential to validate these theoretically established results by performing experimental tests.

\section{REFERENCES}

[1] B. D. Steinberg, Principles of Aperture and Array System Design: Including Random and Adaptive Arrays. New York, Wiley-Interscience, p. 374, 1976.

[2] G. Bradfield, "Improvements in ultrasonic flaw detection," J. Br. Inst. Radio Eng., vol. 14, no. 7, pp. 303-308, 1954.

[3] J. C. Somer, "Electronic sector scanning for ultrasonic diagnosis," Ultrasonics, vol. 6, no. 3, pp. 153-159, 1968. https://www.sciencedirect. com/science/article/abs/pii/0041624X68902771.

[4] R. P. Martin, H. Rakowski, J. French, and R. L. Popp, "Localization of pericardial effusion with wide angle phased array echocardiography," Am. J. Cardiol., vol. 42, no. 6, pp. 904-912, 1978. https://www.ajconline.org/article/0002-9149(78)90674-4/fulltext.

[5] D. K. Lemon and G. J. Posakony, "Linear array technology in NDE applications," Mater. Eval., vol. 38, no. 7, pp. 34-37, 1980.

[6] A. McNab and M. J. Campbell, "Ultrasonic phased arrays for nondestructive testing," NDT Inter., vol. 20, no. 6, pp. 333-337, 1987. https://www.sciencedirect.com/science/article/abs/pii/030 8912687902902.
[7] A. Mariam, A. Khamlichi, and F. El Khannoussi, "Optimizing the focal laws of ultrasonic arrays for non-destructive evaluation of composite panels with delamination," MATEC Web of Conferences, vol. 191. EDP Sciences, 2018. https://www.matecconferences.org/articles/matecconf/abs/2018/50/matecconf_ ndecs2017_00001/matecconf_ndecs2017_00001.html.

[8] W. Shi-Chang and Y. Shi, "Optimum beam steering of linear phased arrays," Wave Motion, vol. 29, no. 3, pp. 245-265, 1999. https://www.infona.pl/resource/bwmeta1.element.elsevier4e120243-c145-39c8-b09a-08231c73078e\#.

[9] W. Shi-Chang and Y. Shi, "Optimization of ultrasonic phased arrays," in Review of Progress in Quantitative Nondestructive Evaluation, D. O. Thompson and D. E. Chimenti, Eds., Boston, USA, Springer, 1998. https://link.springer.com/chapter/10.1007/ 978-1-4615-5339-7_114.

[10] W. Shi-Chang and Y. Shi, "Influence of phased array element size on beam steering behavior," Ultrasonics, vol. 36, no. 6, pp. 737749, 1998. https://www.sciencedirect.com/science/article/abs/pii/ S0041624X97001649.

[11] W. Shi-Chang and Y. Shi, "Three-dimensional beam directivity of phase-steered ultrasound," J. Acoust. Soc. Am., vol. 105, no. 6, pp. 3275-3282, 1999. https://asa.scitation.org/doi/abs/10.1121/1. 424655.

[12] L. Rayleigh, Theory of Sound, Vol. II, Sec. 278. New York, Dover, 1965. (first edition printed in 1877).

[13] L. W. Schmerr, Fundamentals of Ultrasonic Nondestructive Evaluation - A Modeling Approach. New York and London, Plenum Press, 1998. https://www.springer.com/gp/book/9783319304618.

[14] J. J. Wen and M. A. Breazeale, "A diffraction beam field expressed as the superposition of Gaussian beams," J. Acoust. Soc. Am., vol. 83, no. 5, pp. 1752-1756, 1988. https://asa.scitation.org/doi/10. $1121 / 1.396508$.

[15] S. Lester and J.-S. Song, Ultrasonic Nondestructive Evaluation Systems, Springer Science+ Business Media, LLC, 2007. https:// www.springer.com/gp/book/9780387490618.

[16] D. Desheng, Y. Zhang, and J. Liu, "Some extensions of the Gaussian beam expansion: Radiation fields of the rectangular and the elliptical transducer," J. Acoust. Soc. Am., vol. 113, no. 6, pp. 3043-3048, 2003. https://asa.scitation.org/doi/10.1121/1. 1572144.

[17] P. Joon Soo, S. J. Song, and H. J. Kim, "Calculation of radiation beam field from phased array ultrasonic transducers using expanded multi-Gaussian beam model," in Solid State Phenomena, vol. 110. Trans Tech Publications, 2006. https://www.scientific. net/SSP.110.163.

[18] H. Ruiju, L. W. Schmerr, and A. Sedov, "Modeling the radiation of ultrasonic phased-array transducers with Gaussian beams," IEEE Trans. Ultrason. Ferroelectr. Freq. Control, vol. 55, no. 12, pp. 2692-2702, 2008. https://ieeexplore.ieee.org/document/4683476.

[19] Z. Xinyu and T. Gang, "Nonparaxial multi-Gaussian beam models and measurement models for phased array transducers," Ultrasonics, vol. 49.1, pp. 126-130, 2009. https://www.sciencedirect. com/science/article/abs/pii/S0041624X08001340.

[20] W. Tiansi, C. Zhang, A. Aleksov, I. Salama, and A. Kar, "Twodimensional analytic modeling of acoustic diffraction for ultrasonic beam steering by phased array transducers," Ultrasonics, vol. 76, pp. 35-43, 2017. https://www.sciencedirect.com/science/ article/abs/pii/S0041624X1630347X. 
[21] T. Wang, C. Zhang, A. Aleksov, I. Salama, and A. Kar, "Two-dimensional refractive index modulation by phased array transducers in acousto-optic deflectors," Appl. Opt., vol. 56, no. 3, pp. 688-694, 2017. https://www.osapublishing.org/ao/abstract.cfm?uri=ao-56-3-688.

[22] F. Guoxiang, J. Li, and C. Wang, "Design and analysis of MEMS linear phased array," Micromachines, vol. 7.1, p. 8, 2016. https:// www.mdpi.com/2072-666X/7/1/8.

[23] L. Yipeng and D. A. Horsley, "Modeling, fabrication, and characterization of piezoelectric micromachined ultrasonic transducer arrays based on cavity SOI wafers," J. Microelectromech. S., vol. 24, no. 4, pp. 1142-1149, 2015. https://ieeexplore.ieee.org/document/ 7014229 .
[24] Z. Wang, X. Cui, H. Ma, Y. Kang, and Z. Deng, "Effect of surface roughness on ultrasonic testing of back-surface micro-cracks," Appl. Sci., vol. 8, no. 1233, pp. 1-15, 2018. https://www.mdpi.com/ 2076-3417/8/8/1233.

[25] L. Robert Michael and V. Torczon, "Pattern search algorithms for bound constrained minimization," SIAM J. Optimiz., vol. 9, no. 4, pp. 1082-1099, 1999. https://epubs.siam.org/doi/abs/10.1137/ S1052623496300507.

[26] L. Joon-Hyun and S.-W. Choi, "A parametric study of ultrasonic beam profiles for a linear phased array transducer," IEEE Trans. Ultrason. Ferroelectr. Freq. Control, vol. 47, no. 3, pp. 644-650, 2000. https://ieeexplore.ieee.org/document/842052. 